\title{
Colicine types of Shigella sonnei isolated in Hong Kong
}

\author{
C. H. CHAN-TEOH, W. T. WONG, C. T. HUANG, AND H. SHUM
}

From the Department of Microbiology, University of Hong Kong, and the Medical and Health Department $\odot$ Institute of Pathology, Hong Kong

SYNOPSIS Two-hundred and twenty-five strains of Shigella sonnei isolated from sporadic cases of? bacillary dysentery were subjected to colicine typing. The result showed that types 15 and 12 were the $\_$ prevailing types in Hong Kong and two new types which did not fit in with the colicine patterns of the current types were observed. A marked increase in the numbers of strains concurrently resistant to chloramphenicol, tetracycline, and streptomycin occurred in recent years. However, there wase no correlation between drug resistance or biochemical pattern and colicine types. Consistency of colicinogenicity was confirmed by the fact that even a rough variant of a smooth strain had noto changed its colicine pattern.

Abbott and Shannon (1958) reported that colicine production can be used as an epidemiological marker of Shigella sonnei. Since then this method has been widely used for typing of this organism in epidemiological studies. Gillies (1964) and Gillies and Brown (1966) reported respectively two new colicine types (types 14 and 15) and Aoki, Naito, Fujise, Muira, Iwanga, Ikeda, Jinnouchi, Marino, Miyahara, Motoki, and Tokiwa (1969) added another four new indicator strains which were derived from variants of Escherichia coli $\mathrm{K}-12$ strain. Thus, the colicine typing of Sh. sonnei could be brought on to a more precise basis.

Though Huang and Chan-Teoh (1965) reported the incidence of shigella infection in Hong Kong, they made no reference to the colicine types of $S h$. sonnei in their findings. Because of the increased incidence of its infection over other Shigella species in the Far East in recent years (Aoki et al, 1969) and because of the growing interest in its epidemiology, we studied the colicine types as well as the drug resistance and sugar-fermentation of 225 strains of Sh. sonnei collected in Hong Kong between the years 1965 and 1969. The strains included those isolated from patients admitted to the Queen Mary Hospital and those submitted to the Government Pathological Institute, the public health laboratory of the colony, for examination. The results of our findings are presented in this report.

Received for publication 26 November 1970.

\section{Materials and Methods}

SOURCES OF STRAINS

All strains of Sh. sonnei were isolated from stools of rectal swabs of patients of sporadic cases of bacillary? dysentery. Only one representative strain of each? outbreak of bacillary dysentery was included and $\vec{B}$ those isolated from contacts were not accounted for. All, except six strains, were derived from clinica cases with direct complaints of gastroenteritis and. diarrhoea. The six primarily non-dysenteric patients were admitted to hospital for appendicitis ( 2 cases) Hirschsprung's disease (2 cases), retroperitonea tumour ( 1 case), and kidney transplant (1 case) They developed dysentery after admission.

All cultures conformed to the characterization of Sh. sonnei as described by Carpenter (1968), ie, theY were Gram-negative, non-motile rods, fermente glucose and mannitol but were late lactose an sucrose fermenters, and were indole, urease, $\mathrm{H}_{2} \mathrm{~S}_{\text {N }}$ and phenylpyruvic reactions negative. They aggluti nated with specific serum to high titres.

When not in use, the cultures were preserved in $0.3 \%$ semisolid nutrient agar stabs overlaid with thin layer of sterile liquid paraffin. Lyophilize $\$$ cultures of the strains were also kept for reference.

INDICATOR STRAINS FOR COLICINE TYPING The 19 indicator strains were $56,17,56 / 56,2, \mathrm{R} \sigma_{0}$ M19, 2/7, R5, Row, Row/E, Row/I, K-12-30/1 蛋 Row/K, 2/15, 2/64, 56/98, 38, 2M, and R1. Among 636 
these, Row/E, Row/I, Row/K and K-12-30/I were mutants of $E$. coli $\mathrm{K}-12$. More patterns could be obtained if K-12 mutants series was included (Aoki et al, 1969).

\section{DRUG SENSITIVITY TEST}

Oxoid Multodisks incorporated with different antibiotics and chemical agents were employed. The drugs used and their amount per disc are given in Table III. DST agar (Oxoid) was the medium used for sensitivity test.

\section{SUGAR FERMENTATION REACTIONS}

Sugar-fermentation activities were tested in peptone broth containing $1 \%$ of the sugars. Screw-capped bottles were used as containers so that the cultures could be kept for a long time with little evaporation. The final results of negative reaction were read after two months' incubation at $37^{\circ} \mathrm{C}$.

\section{Results}

The results of colicine typing of 225 strains of $S h$. sonnei are presented in Table $I$ and the distribution of the types in Table II. As is seen, the prevalent colicine types of Sh. sonnei in Hong Kong were types $15(43.1 \%)$ and $12(24.9 \%)$, followed by types $16(9 \cdot 6 \%), 8(7 \cdot 7 \%), 9 a(7 \cdot 1 \%), 3 a(3 \cdot 1 \%)$, and $6(1 \cdot 5 \%)$. Types 3,11 , and 17 constituted $1 \%$ each

\begin{tabular}{|c|c|c|c|}
\hline Colicine Type & $\begin{array}{l}\text { No. of } \\
\text { Strains }\end{array}$ & $\begin{array}{l}\text { Percentage of } \\
\text { All Strains }\end{array}$ & $\begin{array}{l}\text { Percentage of } \\
\text { Typable Strains }\end{array}$ \\
\hline $1 \mathrm{a}$ & - & - & - \\
\hline 16 & - & - & - \\
\hline 2 & - & - & - \\
\hline 3 & 2 & 0.9 & 1.0 \\
\hline $3 \mathbf{a}$ & 6 & $2 \cdot 7$ & $3 \cdot 1$ \\
\hline 4 & - & - & - \\
\hline $4 a$ & - & - & - \\
\hline 5 & - & - & - \\
\hline 6 & 3 & $1 \cdot 3$ & 1.5 \\
\hline 7 & - & - & - \\
\hline 8 & 15 & 6.8 & $7 \cdot 7$ \\
\hline 9 & - & - & - \\
\hline $9 a$ & 14 & $6 \cdot 2$ & $7 \cdot 1$ \\
\hline 10 & - & - & - \\
\hline 11 & 2 & 0.9 & 1.0 \\
\hline 12 & 49 & $21 \cdot 7$ & 24.9 \\
\hline 13 & - & - & - \\
\hline $13 a$ & - & - & - \\
\hline 14 & - & - & - \\
\hline 15 & 85 & $37 \cdot 8$ & $43 \cdot 1$ \\
\hline 16 & 19 & 8.4 & 9.6 \\
\hline 17 & 2 & 0.9 & 1.0 \\
\hline 0 & 28 & $12 \cdot 4$ & - \\
\hline
\end{tabular}

Table II Distribution of colicine types among 225 strains of Sh. sonnie isolated in Hong Kong

only. The number of non-typable strains (type 0 ) was high. They accounted for $12.4 \%$.

Types 16 and 17 were new types which did not fit in with the patterns described by previous workers (Abbott and Shannon, 1958; Gillies and Brown, 1966; Aoki et al, 1969). In general, the colicine

\begin{tabular}{|c|c|c|c|c|c|c|c|c|c|c|c|}
\hline \multirow{2}{*}{$\begin{array}{l}\text { Indicator } \\
\text { Strains }\end{array}$} & \multicolumn{11}{|c|}{ Colicine Type } \\
\hline & 3 & $3 a$ & 6 & 8 & $9 a$ & 11 & 12 & 15 & 16 & 17 & 0 \\
\hline 56 & + & + & + & - & + & - & + & - & + & + & - \\
\hline 17 & + & + & - & + & + & - & - & + & - & - & - \\
\hline $56 / 56$ & + & + & + & - & + & - & + & - & + & + & - \\
\hline 2 & + & + & - & + & + & - & + & + & + & + & - \\
\hline R6 & + & + & + & - & + & - & + & - & + & + & - \\
\hline M19 & + & - & - & - & - & - & - & - & - & - & - \\
\hline $2 / 7$ & + & + & - & - & - & - & - & + & + & + & - \\
\hline RS & + & + & - & $t^{2}$ & $t^{2}$ & - & $t^{1}$ & + & + & + & - \\
\hline Row & + & + & + & + & + & + & + & + & + & + & - \\
\hline Row/E & -1 & + & - & - & - & + & - & - & - & + & - \\
\hline Row/I & + & + & + & + & + & + & + & + & + & + & - \\
\hline $\mathrm{K}-12-30 / 1$ & + & + & - & + & + & + & + & + & + & + & - \\
\hline Row/K & + & + & $+^{1}$ & + & + & + & + & + & + & + & - \\
\hline $2 / 15$ & + & + & - & + & + & - & + & + & + & + & - \\
\hline $2 / 64$ & -1 & -1 & - & - & - & - & - & - & - & - & - \\
\hline $56 / 98$ & + & + & + & - & + & - & + & - & + & - & - \\
\hline 38 & + & + & $+^{2}$ & + & + & + & + & + & + & + & - \\
\hline $2 \mathrm{M}$ & + & - & $+1-$ & - & - & - & - & + & + & + & - \\
\hline $\mathbf{R} 1$ & + & + & + & + & + & +1 & + & + & + & + & - \\
\hline $\begin{array}{l}\text { Total no. of } \\
\text { strains }\end{array}$ & 2 & 6 & 3 & 15 & 14 & 2 & 49 & 85 & 19 & 2 & 28 \\
\hline
\end{tabular}

Table I Colicine types of 225 strains of Sh. sonnei isolated in Hong Kong

'Do not conform with the findings of Gillies and Brown (1966) or of Aoki et al (1969). 
patterns of our types agreed with those described in the literature with certain discrepancies. For example, the inhibition of the indicator strain R5 by types $8,9 \mathrm{a}$, and 12 , and of indicator strain 38 by type 6 , and $\mathrm{R} 1$ by type 11 has not been reported before. These variations occurred in all corresponding colicine types in our study. The occurrence of such variations might be due to transference of colicine factor from local strains of $E$. coli as discussed by Farrant and Tomlinson (1966).

The results of drug sensitivity tests are presented in Table III. Only $2 \cdot 2 \%$ of the strains were sensitive to sulphamethoxazole but $94.7 \%$ were sensitive to

\begin{tabular}{llll}
\hline Antibiotic & $\begin{array}{l}\text { Amount of } \\
\text { Drug/Disk } \\
(\mu \mathrm{g})\end{array}$ & $\begin{array}{l}\text { No. of } \\
\text { Sensitive } \\
\text { Strains }\end{array}$ & $\begin{array}{l}\text { Percentage of } \\
\text { Sensitive } \\
\text { Strains }\end{array}$ \\
\hline Chloramphenicol & 10 & 36 & 16.0 \\
Nitrofurantoin & 50 & 211 & $93 \cdot 8$ \\
Nalidixic acid & 30 & 210 & $93 \cdot 3$ \\
Ampicillin & 25 & 200 & 88.9 \\
Streptomycin & 10 & 36 & 16.0 \\
Tetracycline & 10 & 42 & $18 \cdot 7$ \\
Septrin & 25 & 213 & $94 \cdot 7$ \\
Gentamicin & 10 & 217 & 96.4 \\
Sulphamethoxazole & $23 \cdot 75$ & 5 & $2 \cdot 2$ \\
Neomycin & 10 & 225 & 100.0 \\
Kanamycin & 10 & 225 & 100.0 \\
Polymyxin B & 100 & 225 & 100.0 \\
\hline
\end{tabular}

Table III Antibiotic sensitivity of 225 strains of $\mathrm{Sh}$. sonnei isolated in Hong Kong

Septrin (Burroughs and Wellcome) which is trimethoprim and sulphamethoxazole combined. More than $80 \%$ of the strains were resistant to chloramphenicol (C), tetracycline (T), or streptomycin (S). Of these, 158 out of 225 strains $(70 \cdot 2 \%)$ carried a CTS resistance pattern, ie, they were resistant to these three drugs at the same time. Most of the strains were sensitive to ampicillin, nalidixic acid and gentamicin, and all strains were sensitive to kanamycin, neomycin, and polymyxin B. Table IV compares the drug resistance of the strains tested in

\begin{tabular}{|c|c|c|}
\hline & \multicolumn{2}{|l|}{ Period } \\
\hline & $1959-64$ & $1968-69$ \\
\hline & 171 Strains & 225 Strains \\
\hline & $\begin{array}{l}\text { Percentage of } \\
\text { Resistant Strain }\end{array}$ & $\begin{array}{l}\text { Percentage of } \\
\text { Resistant }\end{array}$ \\
\hline Chloramphenicol & 30 & 84 \\
\hline Tetracycline & 28 & 81 \\
\hline Streptomycin & 39 & 84 \\
\hline Nitrofurantoin & 4 & 6 \\
\hline Polymyxin B & 7 & $\mathbf{0}$ \\
\hline Neomycin & 0 & 0 \\
\hline Kanamycin & 0 & 0 \\
\hline
\end{tabular}

Table IV Comparison of drug resistance of Sh. sonnei isolated in two periods the present series and those in a previous study. There $\stackrel{\stackrel{0}{*}}{\circ}$ was no correlation between colicine types and drug resistance patterns.

Of 150 strains tested, $119(79 \cdot 3 \%)$ fermented melibiose in two days, $124(82.6 \%)$ fermented raffinose in 10 days, $144(96.0 \%)$ fermented rhamnose $\overline{\overline{0}}$ in 24 hours, but only $18(12.0 \%)$ fermented xylose $\widehat{\circ}$ within two months (Table V). There was no correlation between biochemical reactions and the $e^{\infty}$ colicine types either. Of the 18 xylose-fermenting. strains, six belonged to type 12,11 to type 16 , and $\overrightarrow{.}$ one to type 0 . Our findings verified those of Aoki et al (1969) who found that colicine type 12 showedo various fermentation and that xylose-positive types? prevailed in Asia. Since all our strains were collected + from sporadic cases of bacillary dysentery the result: does not imply that drug resistance patterns ande్ biochemical types are not useful as subsidiaryo markers for epidemiological study of strains isolated ${ }_{-}$ from outbreaks.

\begin{tabular}{|c|c|c|c|c|c|}
\hline \multirow{2}{*}{$\begin{array}{l}\text { Colicine } \\
\text { Type }\end{array}$} & \multirow{2}{*}{$\begin{array}{l}\text { No. of } \\
\text { Strains } \\
\text { Examined }\end{array}$} & \multicolumn{4}{|c|}{ No. of Strains Fermented } \\
\hline & & Melibiose & Raffinose & Rhamnose & Xylose \\
\hline $\begin{array}{c}3 \\
3 \mathrm{a} \\
6 \\
8 \\
9 \mathrm{a} \\
11 \\
12 \\
15 \\
16 \\
17 \\
0\end{array}$ & $\begin{array}{r}2 \\
4 \\
2 \\
10 \\
15 \\
1 \\
19 \\
58 \\
17 \\
1 \\
21\end{array}$ & $\begin{array}{r}1 \\
2 \\
2 \\
10 \\
15 \\
1 \\
8 \\
58 \\
3 \\
1 \\
18\end{array}$ & $\begin{array}{r}1 \\
3 \\
2 \\
9 \\
14 \\
1 \\
12 \\
58 \\
8 \\
1 \\
15\end{array}$ & $\begin{array}{r}2 \\
2 \\
2 \\
10 \\
15 \\
1 \\
19 \\
58 \\
17 \\
1 \\
17\end{array}$ & $\begin{array}{r}0 \\
0 \\
0 \\
0 \\
0 \\
0 \\
6 \\
0 \\
11 \\
0 \\
1\end{array}$ \\
\hline $\begin{array}{l}\text { Total } \\
\text { Percentage } \\
\text { of strains }\end{array}$ & 150 & $\begin{array}{l}119 \\
79 \cdot 3\end{array}$ & $\begin{array}{l}124 \\
82 \cdot 6\end{array}$ & $\begin{array}{l}144 \\
96 \cdot 0\end{array}$ & $\begin{array}{l}18 \\
12 \cdot 0\end{array}$ \\
\hline
\end{tabular}

Table V Fermentation reactions of 150 strains of Sh. sonneib

Our results also conformed with the observation of Gillies (1964) on the consistency of colicines patterns of Sh. sonnei strains. Rough progenies consistently produced the same colicine pattern of a? smooth parent strain after long periods of stocking

\section{Discussion}

The usefulness of colicine typing as a tool for epi demiological study of Sh. sonnei infection is generalli recognized. Aoki et al (1969) added four indicators? of $E$. coli K-12 mutant origin for typing thus? enabling a clear distinction to be made between types 6 and 11 and types 4 and 14 . They also estab

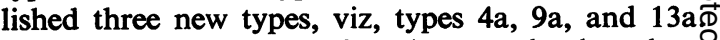
Using their scheme we found not only that there्षे were discrepancies existing in the colicine patterns of 


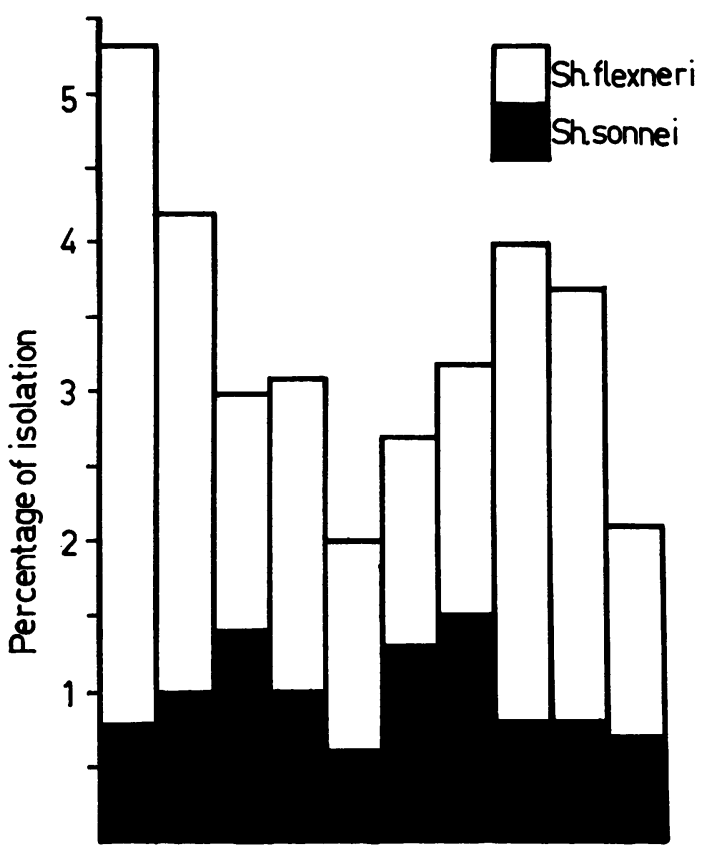

Year 1960196119621963196419651966196719681969

Fig. 1 Percentage incidence of Sh. flexneri and Sh. sonnei isolated from stools.

our strains when compared with the known types, but also two new types (types 16 and 17) which did not fit in with the patterns of the types described by previous workers. Their existence was probably due to the natural occurrence of such variants of $S h$. sonnei strains in Hong Kong or to mutation resulting from transference of colicine factor as indicated by Farrant and Tomlinson (1966).

In Japan the incidence of Sh. sonnei infection has increased and is responsible for more than half of the dysentery cases in large cities in that country. The increase in frequency of Sh. sonnei infection has also been felt in other Far Eastern countries (Aoki et al, 1969). Although there was an appreciable increase in prevalence of $S h$. sonnei infection in Hong Kong, it has not superseded Sh. flexneri in prevalence (Figure 1). It must be noted that our strains were those isolated from selected clinical and hospital groups and they do not actually reflect the true picture in the general population, for Sh. sonnei often produces a milder infection with transient diarrhoea, and asymptomatic cases may even be encountered. Infection of these types usually escapes attention whereas in Japan, when shigellae are found in the stools, despite the absence of clinical symptoms, the law requires that the person must be isolated (Aoki, 1968). The rate of reported cases is, therefore, higher.

Aoki et al (1969) reported that types 6 and 14 were the predominant colicine types of Sh. sonnei isolated in Japan, whereas types 8,6 , and 12 were predominant in Taiwan. In our findings, types 15 and 12 were the most prevalent in Hong Kong. The people in Taiwan and in Hong Kong are largely Chinese and the difference in prevalence of the colicine types appears to be related to the locality rather than to the ethnic group of the people. It must be noted that types 2, 4, and 7, which were frequently isolated in Britain, have not been found in the Far East. With traffic by air becoming heavier, the new types may be introduced from one geographical area to another in future.

Gillies (1964) reported that there was no evidence of change in colicine production either quantitatively or qualitatively when cultures were checked at intervals of six to 12 months over a period of four years. Naito, Kono, Fujise, Yakushiji, and Aoki (1966) found that among 76 strains of Sh. sonnei examined, only one strain changed its colicinogenic activity and five had changed in their sensitivity to colicine after storage. We confirmed the findings of Gillies and found that even rough variants of a strain maintained the colicinogenicity of a smooth parent strain. The stability of colicinogenicity is therefore regarded as a practical and well grounded method of identification of epidemic strains of Sh. sonnei.

The drug resistance of Sh. sonnei isolated in Hong Kong has been described by Huang and Chan-Teoh (1965). There was no change in the resistance patterns except that there was an increase in the percentage of strains resistant to chloramphenicol, tetracycline, and streptomycin combined. The incidence rose from $23.3 \%$ as reported in 1965 to $70.2 \%$ in the present series. This phenomenon was also observed in Japan and other Asian countries, and is probably due to transferable resistance in vivo from one species of enteric organism to another.

Though there was no correlation between colicine types and drug resistance patterns or between colicine types and biochemical types, colicine typing, when used together with drug resistance patterns and biochemical reactions, can be useful for subsidiary pinning down the source of an outbreak. In a recent outbreak of Sh. sonnei dysentery in an orphanage in Taipo, Hong Kong, all the 49 isolates from clinical and asymptomatic cases were identified as colicine type 15. They were all resistant to chloramphenicol, tetracycline, and streptomycin, but were sensitive to nitrofurantoin, nalidixic acid, and ampicillin. Biochemically, all strains fermented melibiose and rhamnose within $\mathbf{4 8}$ hours and raffinose within 10 
days, but xylose was negative. The source of the outbreak was finally traced to the water supply of the orphanage because identical strains were isolated from a stream which supplied the water to the orphanage and a little girl who lived in the neighbouring area had polluted the water.

The problem remains of the presence of the 'unclassifiable' or type 0 strains. Since their colicine patterns are not known, the significance of colicine typing for epidemiological study of these strains is lost. However, when more indicator strains are found in the future, and further attempts are made by combining their colicinogenic activity against the indicators and their sensitivity to colicine produced by the indicators (Naito et al, 1966) the numbers of unclassified strains can then be narrowed down. When compared with those reported in Britain or in Japan, the percentage of type 0 strains in our series $(12.4 \%)$ was unusually high. The high rate of isolation was perhaps due to our selection of sporadic cases. If all strains isolated from outbreaks were included, then the percentage of non-typable strains will be reduced.

We are most grateful to Professor Y. Aoki of the Nagasaki University Medical School for kindly supplying the whole set of indicator strains for our study and to the Director of Medical and Health $\overrightarrow{\bar{\sigma}}$ Services for permission to publish the data of the Institute of Pathology.

References

Abbott, J. D., and Shannon, R. (1958). A method for typing Shigella sonnei, using colicine production as a marker. J. clin. Path., 11,, $\mathbb{\Phi}$ 71-77.

Aoki, Y. (1968). Serological groups of Shigella in Japan and neigh- ָص bouring countries: a review. Trop. Med. Nagasaki, 10, 116-126.

Aoki, Y., Naito, T., Fujise, N., Muira, K., Iwanaga, Y., Ikeda, A. $\overrightarrow{0}$ Jinnouchi, K., Marino, T., Miyahara, A., Motoki, Y., and Tokiwa, H. (1969). Colicin type, biochemical type and drugresistance pattern of Shigella sonnei isolated in Japan and its neighbouring countries: a detailed report. Trop. Med. Naga - S saki, 11, 57-75.

Carpenter, K. P. (1968). Identification of Shigella. Ass. Clin. Path., Broadsheets, 60.

Farrant, W. N., and Tomlinson, A. J. H. (1966). Some studies on the epidemiology of Sonne dysentery. Changes in colicine type and antibiotic resistance between 1956 and 1965. J. Hyg. (Lond.), $\mathrm{W}$
$64,287-303$.

Gillies, R. R. (1964). Colicine production as an epidemiologicalo marker of Shigella sonnei. J. Hyg. (Lond.), 62, 1-9.

Gillies, R. R., and Brown, D. O. (1966). A new colicine type (type 15) of Shigella sonnei. J. Hyg. (Lond), 64, 305-308.

Huang, C. T., and Chan-Teoh, C. H. (1965). Shigella species isolated in Hong Kong with special reference to drug-resistance of strains. Endem. Dis. Bull. Nagasaki, 7, 64-70.

Naito, T., Kono, M., Fujise, N., Yakushiji, Y., and Aoki, Y. (1966). Colicine typing of Shigella sonnei. I. Principle, technique, $\vec{\theta}$ selection of indicator strains, and foundations for a typing scheme. Japan J. Microbiol., 10 13-22.

Shum, H., Sum, C. Y., and Chan-Teoh, C. H. (1971). Water-borne dysentery due to Shigella sonnei in Hong Kong. S.E. Asian J.O trop. Med. and Publ. Hlth, 2, 180-185. 